\title{
ATENDIMENTO DE SAÚDE A UM GRUPO DE IDOSOS CADASTRADOS EM UMA CLÍNICA UNIVERSITÁRIA
}

\author{
Aline Neves OLIVEIRA ${ }^{1}$ \\ Leandra Garcia de M. FONSECA ${ }^{2}$ \\ Patrícia Alves P.CARNEIRO ${ }^{3}$ \\ Zélia Marilda Rodrigues RESCK ${ }^{4}$ \\ ${ }^{1}$ Enfermeira. Mestranda pelo Programa de Pós-graduação em Enfermagem da UNIFAL/MG. E-mail: \\ aline.neoliveira@gmail.com; \\ ${ }^{2}$ Enfermeira. Especialista em Cardiologia pelo UNIS/MG. E-mail:leandra.garcia@ig.com.br; \\ ${ }^{3}$ Mestre em Enfermagem. Coordenadora do Curso de Enfermagem do UNIS/MG. E-mail: \\ patriciaapcarneiro@yahoo.com.br; \\ ${ }^{4}$ Doutora em Enfermagem. Docente do Programa de Pós-graduação em Enfermagem da UNIFAL/MG. E-mail: \\ zeliar@unifal-mg.edu.br.
}

Recebido em: 30/05/2014 - Aprovado em: 25/09/2014 - Disponibilizado em: 15/12/2014

\begin{abstract}
RESUMO
A transição demográfica, vivenciada no Brasil apresenta aos serviços de saúde um importante desafio, já que o aumento da expectativa de vida acarreta maior acometido das condições crônicas em pessoas idosas. Destaca-seo importante papel das universidades, que devem basear a formação emum modelo de atençãoem saúde contrário ao modelo biomédico, incluindoo ensino, a pesquisa e a extensão. Este estudo teve como objetivo descrever e analisar as atividades desenvolvidas em um grupo de idososatendidos em uma Clínica Universitária em uma instituição de ensino no Sul de Minas Gerais.Foi empregadaabordagem qualitativa e, a coleta de dados ocorreuno período de maio a junho de 2011 , através de entrevista semiestruturada. A análise dos dados se deu sob a análise de conteúdo, com elucidaçãode três categorias: "A percepção do Envelhecimento pelo Idoso", "Educação em saúde e mudança no estilo de vida", e "Os grupos e a interação social entre os idosos". Os atendimentos de saúde, oferecidos na clínica, possuem como objetivo auxiliar os idosos no controle às condições crônicas, através da educação em saúde e do trabalho em grupo. Entretanto, o perfil das atividades assistenciais e educativas desenvolvidas pelos professores e acadêmicos, é conservador e pauta-se no modelo de educação e assistência biomédico, com difusão de conhecimentos exclusivos da doença, que não mais atende às necessidades de saúde contemporâneas. Assim, a formação dos futuros profissionais de saúde deve ser refletida, já que as condições sociais, demográficas e epidemiológicas demandam novos paradigmas para o ensino e atuação.
\end{abstract}

Palavras-chave: Saúde do Idoso.Grupo de Idosos. Ensino de Saúde.

\begin{abstract}
The demographictransitionexperienced in Brazilpresentstohealthservicesis a challenge, sincetheincrease in lifeexpectancy leads tohigheraffectedofchronicconditions in theelderly. Highlightstheimportant role ofuniversitiesshould base the training on a modelofcare in contrasttothebiomedicalmodelhealth, includingteaching, researchandextension. Thisstudyaimedtodescribeandanalyzetheactivities in a groupofelderlypatients in a UniversityClinic in aneducationalinstitution in the South of Minas Gerais. Qualitative approach wasusedand data collectionoccurred in theperiod May-June 2011 throughsemi-structured interviews. Data analysisoccurred in thecontentanalysis, withelucidationofthreecategories: "PerceptionsofAgingbyElderly", "Health educationandchange in lifestyle," and "The groupsand social interactionamongtheelderly ". The healthcareservicesofferedattheclinic are aimedathelpingtheelderlytocontrolchronicconditions, throughhealtheducationandgroupwork. However, the profile ofcharitableandeducationalactivitiesdevelopedbyteachersandacademics, isconservativeandisguided educationandbiomedicalcaremodel, withdiffusionof exclusive knowledgeofthedisease, which no longermeetstheneedsofcontemporaryhealth. Thus, the training of future healthprofessionalsshouldbereflected, sincethe social, demographicandepidemiologicalconditionsdemand new paradigms for teachingand performance.
\end{abstract}

Keywords:Aging Health. GroupofElders.Schoolof Health.

\section{INTRODUÇÃO}

A transição demográfica, vivenciada

no Brasil e, no mundo, apresenta aos serviços

de saúde um importante desafio, já que o aumento da expectativa de vida acarreta maior acometido das condições crônicas para as pessoas idosas. No ano de 2007, $72 \%$ das mortes no país foram decorrentes de 
condições como as cardiovasculares, as doenças respiratórias crônicas, o diabetes, os cânceres e as doenças renais. Essa problemática relaciona-se ao aumento da expectativa de vida e de renda, ao desenvolvimento industrial e urbano, ao maior acesso aos alimentos processados e, à globalização (SCHIMIDT et al., 2011).

Nesse sentido, 650 mil novos idosos são incorporados à população brasileira a cada ano, sendo que a maioria apresenta condições crônicas e/ou limitações funcionais. Nos últimos 40 anos, o Brasil passou de um cenário de mortalidade próprio de uma população jovem para um quadro de enfermidades complexas e onerosas, típica dos países mais velhos, caracterizado por doenças crônicas e múltiplas (VERAS, 2009).

Para atender a esta nova demanda, os serviços de saúde devem ter como objetivo principal a manutenção da capacidade funcional do idoso, por meio das ações de promoção da saúde e prevenção de agravos, com vistas a permitir que estepermaneçaativo na sociedade, com maior autonomia e independência para cuidar de si (RAMOS, 2002).

Destaca-se, assim,o importante papel das instituições formadoras de recursos humanos em saúde. As universidades devem direcionar a formação dos alunos para um novo modelo de atenção em saúde, contrário ao modelo biomédico hegemônico, que priorize a formação integral, por meio de atividades de ensino, pesquisa e extensão emetodologias transformadoras que estimulem a reflexão crítica nos alunos (BRASIL, 2001).

Neste sentido, os programas de extensão universitária apresentam-se como estratégias pertinentes a este objetivo, já que promovem o desenvolvimento de importantes relações, entre a instituição de ensino e a sociedade, permitindo a troca de conhecimentos e experiências entre professores, alunos e população, potencializando o processo ensinoaprendizagem a partir de práticas cotidianas. $\mathrm{Na}$ área da saúde, as práticas de extensãoassumem particular relevância, já que estimulam novas experiências voltadas à humanização, ao cuidado e à qualificação da atenção à saúde (HENNINGTON, 2005).

Este estudo teve como objetivo descrever e analisar as atividades desenvolvidas em um grupo de idosos,atendidosem uma Clínica Multidisciplinar Universitária, por acadêmicos e professores dos cursos da área da saúde em uma instituição de ensino no Sul de Minas Gerais, visto que o aumento significativo da população idosa brasileira, assim como na necessidade de desenvolver ações integrais à essa clientela, destaca o papel da universidade enquanto promotora de ações de extensão em saúde, e principal agente de transformação do modelo assistencial de saúde. 


\section{CAMINHO METODOLÓGICO}

A pesquisa qualitativa foi empregada como estratégia metodológica neste estudo, com vistas a conhecer as perspectivas e vivências dos idosos quanto aos atendimentos e intervenções de saúde, realizados em uma Clínica Multidisciplinar Universitária (MATHEUS; FUSTINONI, 2006).

A coleta de dados foi realizadano período de maio a junho de 2011 , por meio da utilização de entrevista semiestruturada e gravação das falas, após assinaturado Termo de Consentimento Livre e Esclarecido.Os participantes foram abordados na própria clínica, durante a realização dos atendimentos e desenvolvimento de suas atividades, perfazendo uma amostra de conveniência.

As questões que nortearam a entrevista foram: "descreva as atividades/atendimentos que o senhor desenvolve/recebe na clínica"; "a sua vida mudou após a participação dos atendimentos? Como?"

A análise dos dados se deu através da Análise de Conteúdo, sob a perspectiva de Minayo (2013), que conta com três etapas inter-relacionadas entre si, sendo elas a Préanálise, com emprego da transcrição do material empírico e exaustivas leituras flutuantes; a Exploração do Material, com classificação e categorização, de acordo com as falas mais frequentes encontradas na préanálise e, por fim, o Tratamento dos Dados, com análise reflexiva dos significados relatados nas entrevistas.
Este estudo foi aprovado pelo comitê de ética em pesquisa do Centro Universitário do Sul de Minas - UNIS/MG, sob parecer 0011/2011 e, seguiu princípios e diretrizes preconizados pela Resolução 466 de 12 de dezembro 2012, do Conselho Nacional de Saúde (CNS).

\section{RESULTADOS}

Participaram do estudo oito idosas do grupo da terceira idade "Viver bem com a idade que você tem". Os integrantes do grupo recebem atendimentos de Fisioterapia, Educação Física, Nutrição e Enfermagem, coordenadas por acadêmicos e professores dos cursos da Saúde, do Centro Universitário do Sul de Minas UNIS/MG.

A clínica foi criadacomo um projeto de extensão, com objetivo de proporcionar maior integração entre teoria e prática para os alunos dos cursos da saúde, durante a formação acadêmica. Os atendimentos de saúde oferecidos são gratuitos e destinam-se à toda população do município, fortalecendo a prestação de serviço social pela universidade.

A maioria das participantes deste estudo,se autodeclaroubranca, viúva, aposentada e, com idade entre 61 e 81 anos. As principais condições crônicas declaradas incluíram Hipertensão, Diabetes Mellitus, Cardiopatias, Doenças Musculoesqueléticas e Autoimunes. 


\section{DISCUSSÃO}

Após análise dos dados, foram elucidadas três categorias: "A percepção do Envelhecimento pelo Idoso", "Educação em saúde e mudança no estilo de vida", e por fim, "Os grupos e a interação social entre os idosos".

As falas dos participantes foram indicadas pela letra "I", seguida de sequência alfanumérica.

\section{A Percepção do Envelhecimento pelo idoso}

O processo de envelhecimento é singular e vivenciado unicamente por cada pessoa. Assim, as perspectivas se baseiam nas experiências de vida, no meio social em que se insere, bem como no apoio que recebe da sociedade, dos serviços de saúde e da família nesta fase da vida (CARVALHO; ROCHA; LEITE, 2002).

O envelhecimento deve ser compreendido como um processo natural, em que há diminuição progressiva da reserva funcional dos indivíduos. Quando este processo ocorre sem a apresentação de problemas de saúde é denominado Senescência, entretanto, na presença de doenças ou agravos à saúde, o processo denomina-se Senilidade. Esses efeitos podem ser minimizados pela assimilação de um estilo de vida mais ativo, bem comopela otimização do acesso aos bens e serviços de saúde (BRASIL, 2006).

As dificuldades enfrentadas durante o envelhecimento relacionam-se ao desenvolvimento das condições crônicas, querefletemem necessidades bastante variadas. Estas condições devem ser amplamente compreendidascom vistas a auxiliar o idoso a nos modos de enxergar o envelhecimento e a velhice, tanto nos aspectos individuais quanto coletivos, (SILVA, 2008).

Para tanto, é necessário apreender o conceito de Envelhecimento Ativo, caracterizado como o "processo de otimização das oportunidades de saúde, participação e segurança, com o objetivo de melhorar a qualidade de vida à medida que as pessoas ficam mais velhas" (WHO, 2005), com o propósito de reorientar os modelos de atenção à saúde da pessoa idosa, por meio de ações que estimulem a autonomia e o autocuidado.

As ações de promoção à saúde e prevenção de agravos surgem, então, como o melhor caminho a ser percorrido pelos serviços de saúde. A Promoção é o processo que permite às pessoas controlar e melhorar sua saúde, por meio do acompanhamento e da educação. Já as ações Preventivas abrangem a prevenção agravos e o tratamento de enfermidades, comuns aos indivíduos em processo de envelhecimento (U.S.; 1999). 
Educação em Saúde a as modificações no estilo de vida

A Educação em Saúde é uma valiosa estratégia para as ações de Promoção à saúde, já que abrange a participação de toda a população no contexto de sua vida cotidiana (CASTRO et al., 2009). As práticas educativas encontram-se vinculadas ao exercício da cidadania na busca por melhores condições de vida, promovendo espaços para troca de informação e, permitindo identificar as demandas de saúde dos indivíduos (ARAÚJO, 2004).

Neste sentido, a Educação em Saúde constitui intervenção precípua em saúde, uma vez que não só veicula informações, mas sugere alternativas para a prevenção de doenças e agravos (HORTA et al, 2009). No que se refere às pessoas idosas e suas condições crônicas, as práticas educativas auxiliam para que estas reflitam suas decisões e, desta maneira, optem por escolhas que priorizem a saúde e a qualidade de vida (CANDEIAS, 1997).

Aspráticas educativas desenvolvidas na Clínica Multidisciplinar Universitária, cenário deste estudo, não possuem estratégias específicas para sua operacionalização. Estes momentos acontecem de maneira informal, durante as consultas e atendimentos e, os acadêmicos e professores oferecem informações quanto à alimentação, à importância da prática de atividades físicas, do lazer e da socialização.

(...) eles falam de qualidade de vida, memória... movimentos, mobilidade do corpo (II).

(...) eles me oferecem todas as informações que eu preciso e tudo que eu quero eu pergunto e elas me respondem espontaneamente (...) (I2).

(...) como cuidar do corpo, da alimentação, como cuidar da saúde (...) (I4).

(...) sobre doença, assim osteoporose, problema de coração, de circulação e de sexo (I6).

(...)Eu sou bem esclarecida sobre todos os temas (I6).

(...) sou muito curiosa quanto a tudo que fala sobre o corpo humano e sobre a saúde (...) (I8).

O perfil da Educação em Saúde,oferecida durante os atendimentos da clínica, dialoga com os métodos tradicionais de educação, desenvolvidos nos setores e serviços de atenção. Na maioria das vezes, estas intervenções educativas são fragmentadas e reduzidas, já que abordam apenas o aspecto biológico do processo de adoecimento e empregam metodologias bastante tradicionais, sendo o profissional de saúde detentor de todo o saber científico e, o idoso mero expectador das informações (ALVES; NUNES, 2006).

Esta problemática relaciona-se amplamente ao modelo de formação biomédico hegemônico, pautado no ensino fisiopatológico, tratamentos e cuidados específicos para as doenças, deixando de lado referenciais sociais e culturais. No entanto, compreender os aspectos subjetivos do adoecimento crônico e envelhecimento é fundamental para melhor desenvolver práticas assistenciais em saúde, já que estes imperam 
constantemente a tomada de decisões e hábitos dos indivíduos (ALVES, 2005).

Nesse sentido, Oliveira (2009) afirma que as práticas educativas desenvolvidas nos setores e serviços de saúde devem ser refletidas quanto à sua razão e as metodologias empregadas, bem como considerar os saberes trazidos pelos indivíduos. Além disso deve-se incluir, ainda na graduação, referenciais para a promoção à saúde que vão além dos que consideram apenas os paradigmas biológicos do envelhecer e adoecer, a fim de satisfazer as necessidades de saúde, mesmo em situações desfavoráveis (SOUZA; BONILHA; VERONESE, 2008).

\section{Os grupos e a interação social entre os idosos}

Os grupos de idosos, ou grupos da terceira idade, constituem estratégias importantes para a promoção da saúde. Estes representam tanto um espaço de oferta de informações como uma fonte de estímulo à cidadania e socialização, através da retomada papeis sociais e outras atividades de ocupação do tempo livre. Os grupos contribuem, ainda, para a criação de vínculos entre os profissionais de saúde e os idosos (GARCIA et al., 2006).

É possível afirmar quea interação e o convívio social proporcionam impacto positivo sobre o estado de saúde do idoso, já que o isolamento está diretamente relacionado ao aumento do risco de morbimortalidade. Assim, a socialização e interação em um grupo de pessoas protege o idoso dos efeitos negativos das transições da vida; como a partida dos filhos, a aposentadoria e a viuvez (WETLE, 1995).

Os participantes deste estudo relataram modificações em suas vidas e rotinas, após participarem do grupo "Viver bem com a idade que você tem", que acolhe idosos de todas as regiões do município onde se localiza a instituição de educação-saúde. Nos encontros eles conversam, trocam informações e confidências, criam vínculos de amizade e confiança, aspectos confirmados por meio das falas dos participantes.

(...) minha vida mudou, eu fiquei mais alegrinha, mais animada (I4).

(...) eu gosto muito de turma, de conversar entendeu? (I2).

(...) fiquei conhecendo gente jovem, pessoas dinâmicas (...) (I5).

(...) mudou muito sabe (...) aqui melhorou muito. (I6).

O principal objetivo dos setores e serviços de saúde, ao desenvolverem grupos de pessoas com características comuns, é oferecer intervenções de saúde a várias pessoas ao mesmo tempo. Estratégias educativas incluem a formação de grupos operativos, onde os idosos são os objetos da ação, com o propósito de estimular a mudança de comportamentos e a adoção de hábitos de vida mais saudáveis (VICTOR et al., 2006).

No entanto, muitas vezes, o papel desempenhado pelo grupo na vida do idosos 
vai além do que preconiza seu objetivo inicial. Este momento torna-se privilegiado para o diálogo, para a interação, para as trocas e para o convívio social, tornando o envelhecer permeado pelo sentir-se bem e, ainda, um tempo de novas descobertas e habilidades (VAROTO; TRUZZI; PAVARINI, 2004).

A participação dos idosos em atividades de grupo configuram um aspecto diferencial da assistência em saúde, pois contribui para a vivência plena do bem estar, impulsionando o desenvolvimento de uma nova visão de futuro, transformando-o um processo de construção e, não mais de finitude (SILVA et al., 2003).Nesse sentido, estimular a participação social dos idosos, por meio da criação de grupos, é premissa fundamental para promover saúde e interação na terceira idade.

\section{CONCLUSÕES}

A população idosa cresce substancialmente em detrimento das demais classes populacionais e, o aumento progressivo da longevidade traz as condições crônicas que demandam acompanhamento e controle contínuos, configurando um importante desafio aos serviços de atenção à saúde, assim como às instituições de ensino.

A universidade assume um importante papel no que se refere à formação de recursos humanos em saúde, bem como à transformação dos modelos de atenção à população idosa e, nesse sentido, desenvolver atividades de extensãocom o propósito de articular teoria e prática torna-se fundamental, já que proporciona uma formação próxima à realidade social e de trabalho.

Osatendimentos de saúde possuem objetivo de auxiliar os idosos no controle às condições crônicas, por meio da educação em saúde e do trabalho em grupo. No entanto, o perfildas atividades assistenciais e educativas desenvolvidas na clínica, pelos professores e acadêmicos, ainda é conservador e pauta-se no modelo de educação e assistência biomédico, com difusão de conhecimentos exclusivos da doença e de seus aspectos biológico e,este modelo de formação e assistência não mais atende às necessidades de saúde de idosos portadores de condições crônicas, já que estas são permeadas constantemente pelos aspectos subjetivos, que ditam comportamentos e hábitos nos indivíduos.

Nesse sentido, a formação dos futuros profissionais de saúde deve ser refletida e implementada, já que as condições sócio demográficas e epidemiológicas atuais demandam novos paradigmas para o ensino e atuação. Os alunos de graduação, com formação generalista para a saúde devem ser capazes de entender não apenas a fisiopatologia das doenças e processos vitais, mas também reconhecer a magnitude do processo saúde-doença, objetivando atuar e intervir em saúde de maneira integral, humanizada e segura. 


\section{REFERÊNCIAS}

ALVES, V.S. Um modelo de educação em saúde para o Programa Saúde da Família: pela integralidade da atenção e reorientação do modelo assistencial. Rev. Interface - Comun. Sau. Educ, Bahia, v. 9, n. 16, p.39-52, fev. 2005.

ALVES, V.S.; NUNES, M.O. Educação em Saúde na atenção médica ao paciente com hipertensão arterial no Programa Saúde da Família. Rev. Interf - Comun. Sau.

Educ, Bahia, v. 19, n. 10, p.131-147, jan. 2006.

ARAÚJO F.M. Ações de educação em saúde no planejamento familiar nas unidades de saúde da família no município de Campina Grande- PB [monografia]. Campina Grande (PB): Universidade Estadual da Paraíba; 2004.

BRASIL. Conselho Nacional de Saúde.

Ministério da Saúde. Resolução No 466, de 12 de dezembro de 2012. Revoga as Resoluções CNS Nos 196/96, 303/2000 e 404/2008.

BRASIL. Ministério da Saúde. Caderno de Atenção Básica $N^{\circ} 35$. Estratégias para o cuidado da pessoa com doença crônica. Brasília: Ministério da Saúde, 2014, 162 p.

BRASIL. Ministério da Saúde. Secretaria de Atenção à Saúde Departamento de Atenção Básica. Cadernos de Atenção Básica no 19:
Envelhecimento e Saúde da Pessoa Idosa. Brasília, Df: Ministério da Saúde, 2006. 192 p. BRASIL. Ministério da Saúde. Caderno de Atenção Básica $N^{\circ} 35$. Estratégias para o cuidado da pessoa com doença crônica. Brasília: Ministério da Saúde, 2014, 162 p.

Brasil. Ministério da Educação. Conselho Nacional de Educação, Câmara de Educação Superior. Institui Diretrizes Curriculares Nacionais do Curso de Graduação em Enfermagem [Internet]. Diário Oficial da União, Brasília (DF); 9 de nov de 2001, Seção 1, p. 37. [citado 02 jul. 2013].

CANDEIAS, N.M.F., Conceitos de educação e de promoção em saúde: mudanças individuais e mudanças organizacionais. Rev. Saúde Pública, 31 (2): 209-13, 1997.

CARVALHO, H. B. C.; ROCHA, S. M.; LEITE, M. L. C. B.. A interação do idoso à prática de saúde. In: FREITAS, Elizabete Viana de et al. Tratado de Geriatria e Gerontologia. Rio de Janeiro: Guanabara Koogan, 2002. Cap. 127, p. 1051-1055.

CASTRO, M.M.C. et al. O cuidado ao hipertenso no município de Juiz de Fora/MG: uma análise comparativa em Unidade Básica de Saúde. Rev. APS, v. 12, n. 3, p. 249-261, jul./set. 2009. 
U.S. Departmentof Health andHuman

Services (1999). AnOunceofPrevention.

What Are theReturns? Atlanta: U.S.

Departmentof Health andHuman Services,

Centers for DiseaseControlandPrevention.

GARCIA, M.A.A. et al. Atenção à saúde em grupos sob a perspectiva dos idosos. Rev

Latino-am Enfermagem, Campinas, v. 14, n.

2, p.175-182, 2006.

GOLDIM, J.R. Bioética e envelhecimento. In: FREITAS, Elizabete Viana de et al. Tratado de Geriatria e Gerontologia. Rio de Janeiro: Guanabara Koogan, 2002.

Cap. 9, p. 85-90.

HORTA, N.C. et al. A prática de grupos como ação de promoção da saúde na estratégia saúde da família. Rev APS, v. 12, n. 3, p. 293301, jul./set. 2009.

HENNINGTON, E.A. Acolhimento como prática interdisciplinar num programa de extensão universitária. Rev. Cad. Saúde

Pública, Rio de Janeiro, v. 21, n.1, p:256-265, 2005.

MATHEUS, M.C.C.; FUSTINONI,

S.M. Pesquisa Qualitativa em enfermagem.

São Paulo: Livraria Médica Paulista, 2006.

$164 \mathrm{p}$.
MINAYO, M.C.S. O desafio do

conhecimento: pesquisa qualitativa em saúde. 13. ed. São Paulo: Hucitec, 2013. 407 p.

MINAYO, M.C.S.; COIMBRA JUNIOR, C.E.A.(Orgs). Antropologia, saúde e envelhecimento. Rio de Janeiro: FIOCRUZ, 2002, 209 p.

OLIVEIRA, C.B. et al. As ações de educação em saúde para crianças e adolescentes nas unidades básicas da região de Maruípe no município de Vitória. Rev Ciência \& Saúde Coletiva, n. 2, v. 14, p. 635-644, 2009.

SCHMIDT, M.I. et al. Doenças crônicas não transmissíveis no Brasil: carga e desafios atuais. The Lancet, v. 377, p.1949-1961, 2011.

SILVA, L.R.F. Da velhice à terceira idade: o percurso histórico das identidades atreladas ao processo de envelhecimento. Rev. História, Ciências, Saúde - Manguinhos, Rio de Janeiro, v.15, n.1, p.155-168, 2008.

SILVA, D.G.V. et al. Grupos como possibilidade para desenvolver educação em saúde. Rev. Texto \& contexto enferm, v.12, n1, 2003, p.97-103.

SOUZA, A.C.; BONILHA, A.L.L.;

VERONESE, A.M. Buscando a integralidade no atendimento ao indivíduo diabético: do 
serviço de atendimento móvel de urgência à atenção primária à saúde. Rev Ciência

Cuidado e Saúde, n. 1, v. 7, 2008, p. 021026.

RAMOS, L. R. Epidemiologia do envelhecimento. In: FREITAS,

Elizabete Viana de et al. Tratado de

Geriatria e Gerontologia. Rio de Janeiro:

Guanabara Koogan, 2002. Cap. 7, p. 72-78.

VAROTO, V. A. G; TRUZZI, O. M. T;

PAVARINI, S. C. L. Programa para idosos independentes: um estudo sobre seus egressos e a prevalência de doenças crônicas. Rev.

Texto e Contexto de Enfermagem,

Florianópolis, v. 1, n. 13, 2004, p.107-114.

VERAS, R. Envelhecimento populacional contemporâneo: demandas, desafios e inovações. Rev. Saúde Pública, v. 43, n. 3, p. 548-554, 2009.

VICTOR, J.F. et al. Grupo Feliz Idade:

cuidado de enfermagem para a promoção da saúde na terceira idade. RevEscEnferm USP, n. 41, n. 4, 2007, p.724-730.

WETLE, T. Questões sociais. In: ABRAMS, William B.; BERKOW, Robert. Manual

Merck de Geriatria. 7. ed. São Paulo: Roca, 1995. Cap. 97, p. $1206-1209$.

WHO. World Health Organization.

\section{Envelhecimento ativo: uma política de} saúde. Brasília: Organização Pan-Americana da Saúde, 2005, 60 p. Tradução Suzana Gontijo. 